\title{
Laparoscopic left hepatectomy with tumor thrombectomy in patients with hepatocellular carcinoma concomitant with advanced portal vein tumor thrombus
}

\author{
Shin Nakahira • Yutaka Takeda • Yoshiteru Katsura • \\ Takeshi Kato • Nobutaka Hatanaka • \\ Shigeyuki Tamura
}

Received: 28 May 2014 / Accepted: 2 July 2014/Published online: 24 July 2014

(c) The Author(s) 2014. This article is published with open access at Springerlink.com

\begin{abstract}
Background Although laparoscopic hepato-biliary-pancreatic surgery has been widely adopted, use of laparoscopic resection for hepatocellular carcinoma (HCC) with advanced portal vein tumor thrombus (PVTT) is uncommon because of the complications involved.

Methods From June 2010 through November 2013, 200 laparoscopic hepatectomies were performed. We report the short-term outcome of laparoscopic hepatectomy for HCC with advanced PVTT in 3 patients. Video presentation is a demonstration of the operative procedures employed in Case 3. In this case, the left hepatic artery and left hepatic duct were divided before tumor thrombectomy, and the bifurcation of the portal vein was clearly visible.

Results Three female patients with HCC concomitant with PVTT in the portal trunk or the opposite branch underwent laparoscopic left hepatectomy with tumor thrombectomy using a laparoscopy-assisted technique (1 patient) or pure laparoscopic technique ( 2 patients). The median operative time was 592 min (range, 555-891 min), and median estimated blood loss was $1182 \mathrm{ml}$ (range,
\end{abstract}

This work has been presented at the 2014 EAES congress in paris.

Electronic supplementary material The online version of this article (doi:10.1007/s00464-014-3729-x) contains supplementary material, which is available to authorized users.

S. Nakahira $(\square) \cdot$ N. Hatanaka

Department of Digestive Surgery, National Hospital

Organization Kure Medical Center and Chugoku Cancer Center,

3-1 Aoyama-cho, Kure, Hiroshima 737-0023, Japan

e-mail: snakahira-gi@umin.ac.jp

S. Nakahira $\cdot$ Y. Takeda $\cdot$ Y. Katsura $\cdot$ T. Kato $\cdot$ S. Tamura Department of Digestive Surgery, Kansai Rosai Hospital, Amagasaki, Japan minimal amount $-4800 \mathrm{ml}$ ). The median length of hospital stay was 19 days (range, 9-22 days), and there was no postoperative mortality. In Case 1 , recurrent tumors developed in the residual lobe after curative resection, and the patient died 10 months after the surgery despite treatment with sorafenib and transcatheter arterial chemoembolization. In Case 2, the patient survived for 10 months after curative resection without tumor recurrence. In Case 3 , the patient was treated with sorafenib 1 month after palliative resection; she survived for 4 postoperative months, during which decreased tumor marker levels were observed.

Conclusions Laparoscopic hepatectomy for HCC with advanced PVTT is a safe and feasible procedure in selected patients, when performed by surgeons with expertise in hepatic surgery and minimally invasive techniques. Although these patients cannot be cured by surgery alone, early adjuvant therapy followed by laparoscopic surgery might contribute to a good outcome.

Keywords Hepatocellular carcinoma P Portal vein tumor thrombus · Laparoscopic hepatectomy · Tumor thrombectomy

Disclosures Drs. Shin Nakahira, Yutaka Takeda, Yoshiteru Katsura, Takeshi Kato, Nobutaka Hatanaka and Shigeyuki Tamura have no conflicts of interest or financial ties to disclose.

Open Access This article is distributed under the terms of the Creative Commons Attribution License which permits any use, distribution, and reproduction in any medium, provided the original author(s) and the source are credited. 